\title{
Cuantificación de ácidos grasos a partir de biomasa microalgal
}

\author{
Por J.L. García Sánchez, E. Molina Grima, F. García Camacho, J.A. Sánchez Pérez y A. Giménez Giménez \\ Departamento de Ingeniería Química. Facultad de Ciencias Experimentales. Universidad de Almería, 04071 Almería.
}

\section{RESUMEN}

Cuantificación de ácidos grasos a partir de biomasa microalgal.

El interés creciente por los ácidos grasos poliinsaturados de cadena larga viene favorecido por la mejora de las técnicas de análisis. La metodología de transesterificación directa propuesta por Lepage y Roy está siendo ampliamente utilizada para la determinación del contenido en ácidos grasos de microalgas, consideradas como potenciales fuentes de estos productos. En el presente trabajo, se analiza la influencia del estado físico de la biomasa analizada, seca, normalmente por liofilización, o húmeda recién cosechada del cultivo. Asimismo, se analiza el efecto de la cantidad de biomasa empleada y del número de extracciones de los ésteres metílicos obtenidos. Del estudio estadístico realizado se pone de manifiesto la importancia del estado físico de la muestra, aconsejándose el uso de biomasa directamente obtenida del cultivo. Se proponen los intervalos de cantidad de biomasa para metilación, así como la conveniencia de realizar tres extracciones previas a la inyección en el cromatógrafo, que favorece la resolución del análisis.

PALABRAS-CLAVE: Acido graso (determinación) - Acido graso poliinsaturado - Estado físico (comparación) - Microalga.

\section{SUMMARY}

Fatty acid determination from microalgal biomass.

Long chain polyunsaturated fatty acids are focusing the interest of researchers as long as analytical techniques are being improved. The Lepage and Roy direct transesterification method is being widely used to measure fatty acid content of microalgae which are proposed as potential sources of these products. In this paper, the physical state of the biomass to be analyzed is studied. Comparison has been stated between lyophilized biomass and wet biomass just harvested. Furthermore, the amount of sample and the number of methyl ester extractions have also been studied. From the statistical analysis carried out, wet biomass to fatty acid determination and the adecuate range of sample are proposed. Three methyl ester extractions improve the resolution of GC analysis.

KEY-WORDS: Fatty acid (determination) - Microalgae - Physical state (comparison) - Polyunsaturated fatty acid.

\section{INTRODUCCION}

Son muchos los trabajos publicados que hacen referencia a la aplicación de los ácidos grasos poliinsaturados extraídos a partir de microalgas, existiendo una amplia bibliografía sobre las especies y condiciones ambientales que favorecen su producción (1) (2) (3).
En la actualidad, parte de las investigaciones en biotecnología de microalgas se conducen a la obtención de cepas con alto contenido en ácidos grasos poliinsaturados, PUFAs (especialmente ácido eicosapentaenoico, EPA, araquidónico, AA, y docosahexaenoico, DHA) y al desarrollo de métodos de extracción y purificación de dichos ácidos orientados a escala industrial (4) (5) (6).

Es necesario, por tanto, disponer de métodos de análisis fiables, sencillos y rápidos de estos ácidos grasos. La técnica instrumental empleada para este propósito es la cromatografía gaseosa, que en los últimos diez años ha experimentado un gran avance con el desarrollo de una gran variedad de columnas y fases estacionarias, dependiendo del perfil de ácidos grasos del material biológico de partida susceptible de análisis (7).

Los métodos más usuales de preparación de las muestras consisten en la extracción de los lípidos y posterior derivatización para obtener los ésteres metílicos de los ácidos grasos (FAMEs), que son los compuestos resueltos por la cromatografía de gases en la mayoría de los casos (8) (9) (10) (11) (12) (13). También en este punto hay una gran variedad de métodos, siendo los más ventajosos aquéllos en los que la derivatización se realiza en una sola etapa (14) (15). Este método ha sido contrastado con otros tres que realizan extracción previa (16), y es la técnica usada en nuestro laboratorio.

En este trabajo se pretende aplicar, con algunas modificaciones, el método de Lepage y Roy (14) a biomasa húmeda de microalgas recién cosechada, y comparar los resultados con biomasa liofilizada, que es el punto de partida de los análisis de ácidos grasos en la mayoria de los trabajos publicados en este campo, independientemente de cuál sea el proceso de esterificación. Esta técnica permitiría conocer el perfil de ácidos grasos de un determinado cultivo dos horas después de la toma de muestra por término medio, así como agilizar los seguimientos de estas sustancias durante el crecimiento del microorganismo. Además, se propone disminuir la cantidad de biomasa para la transesterificación que propone dicho método $(10 \mathrm{mg})$ (14). En consecuencia, para cultivos poco densos (100 y $300 \mathrm{mg} / \mathrm{l})$, con $10 \mathrm{ml}$ del cultivo bastaría para el análisis, y para cultivos con concentraciones mayores el método sería aún más favorable, ya que con 2-3 ml sería suficiente.

La modificación del método con respecto al original consiste en hacer dos extracciones adicionales con el mismo 
volumen ( $1 \mathrm{ml}$ de hexano) y reunirlas para su análisis, comprobando previamente la recuperación que se consigue inyectando las fracciones por separado.

\section{MATERIALES Y METODOS}

Se ha utilizado la microalga marina Isochrysis galbana Parke, con la cual se han realizado diferentes estudios conducentes a determinar la influencia de las condiciones de crecimiento en el perfil de ácidos grasos obtenido, tanto en cultivo discontinuo como continuo (17) (18). Se realizó una recta de calibrado concentración en peso seco vs. densidad óptica a $530 \mathrm{~nm}$.

$$
\mathrm{C}(\mathrm{mg} / \mathrm{l})=\text { D.O. }{ }_{530}{ }^{*} 232 ; \mathrm{r}^{2}=0,9987, \mathrm{n}=15(19) \text {. }
$$

La biomasa utilizada de esta microalga procedía de un cultivo batch en matraz de $1500 \mathrm{ml}$ con una aireación de 1 v.v/min, medio de Ukeles modificado (20) e intensidad de iluminación de $60 \mathrm{~W} / \mathrm{m}^{2}$, alcanzando una concentración de $650 \mathrm{mg} / \mathrm{l}$, en el momento de efectuar los análisis.

El procedimiento experimental seguido fue el siguiente: para los análisis por vía húmeda se centrifugaron varias alicuotas de cultivo para obtener las cantidades de biomasa que se eligieron como intervalo experimental $(1-20 \mathrm{mg})$ y se realizó la transesterificación siguiendo el método de Lepage y Roy (14). La extracción de los ésteres metílicos se realizó con $1 \mathrm{ml}$ y $3 \mathrm{ml}$ de hexano para dos series experimentales, respectivamente. Seguidamente se centrifugó el resto del cultivo y se liofilizó. Finalizado este proceso se homogeneizó la biomasa y se pesaron las muestras para el análisis por vía seca, diferenciando también el volumen de la extracción.

Previo a la inyección en el cromatógrafo, se llevaban a sequedad las fracciones de hexano (reunidas en el caso de realizar tres extracciones), bajo corriente de $\mathrm{N}_{2}$ y se resuspendían en $0,1 \mathrm{ml}$ de hexano.

Para comprobar la extracción total de ácidos grasos en los ensayos con tres extracciones, se realizó el correspon- diente cromatograma a tres muestras de biomasa de 10 , 15 y $20 \mathrm{mg}$ para cada una de las extracciones.

Los análisis se han realizado con un cromatógrafo de gases HP5890 series II equipado con un FID y un integrador HP 3394. Se ha utilizado una columna capilar (Supelco) de sílice fundida de alta polaridad SP2330 de $30 \mathrm{~m}$ de longitud y $0,25 \mathrm{~mm}$ de diámetro interno y 0,2 micras de espesor de film. El flujo de gas portador $\left(\mathrm{N}_{2}\right)$ fue de $0,8 \mathrm{ml} / \mathrm{min}$ y se utilizó el sistema de inyección con división de flujo con una relación de venteo de 100:1. La temperatura del inyector y del detector fue de $220^{\circ} \mathrm{C}$. El programa de temperatura del horno fue $150^{\circ} \mathrm{C}(8 \mathrm{~min})$ con una rampa de $3^{\circ} \mathrm{C} / \mathrm{min}$ hasta $190^{\circ} \mathrm{C}(21 \mathrm{~min})$. El volumen de inyección fue de $2 \mu$, y cada dos análisis se realizaba un blanco. Todas las transesterificaciones se hicieron por duplicado.

El método de integración ha sido el de patrón interno, siendo éste el ácido nonadecanoico, 19:0, añadido a la muestra antes de la transesterificación $(5 \mu$ ) de patrón de concentración $31,25 \mathrm{mg} / \mathrm{ml}$, disuelto en metanol-benceno $3: 2)$. Los patrones para identificación de los ácidos grasos son de Supelco (PUFA $n \div 1$ y PUFA $n^{\circ}$ 2) y los patrones para la mezcla de calibración fueron suministrados por Sigma.

En lo referente al cálculo de los factores de respuesta para el detector, hay pocos trabajos que indiquen el procedimiento. En nuestro caso, se ha hecho una aproximación, que consiste en calcular el factor de respuesta para los ácidos grasos mayoritarios presentes en esta microalga y asignar el mismo valor a los del mismo grupo (igual número de átomos de carbono en la cadena), y obviamente para el patrón, ya que disponer de patrones individuales de cada uno de los ésteres metílicos identificados sería muy costoso, particularmente para los ácidos poliinsaturados.

El análisis estadístico de los datos ha sido realizado por el programa STATGRAPHICS ver. 5 .

Tabla I

Resultados del ajuste por regresión lineal de la cantidad de ácido graso identificado vs cantidad de biomasa metilada

\begin{tabular}{|c|c|c|c|c|c|c|c|c|c|c|c|c|}
\hline \multirow[b]{2}{*}{$\begin{array}{l}\text { Acido } \\
\text { graso }\end{array}$} & \multicolumn{3}{|c|}{$1 \mathrm{ml}$ de hexano $(\mathrm{w})^{\star}$} & \multicolumn{3}{|c|}{$3 \mathrm{ml}$ de hexano $(\mathrm{w})$} & \multicolumn{3}{|c|}{$1 \mathrm{ml}$ de hexano $(\mathrm{d})^{\star}$} & \multicolumn{3}{|c|}{$3 \mathrm{ml}$ de hexano $(\mathrm{d})$} \\
\hline & Oo & $P$ & $r$ & Oo & $\mathrm{P}$ & $r$ & Oo & $\mathrm{P}$ & $r$ & Oo & $P$ & $r$ \\
\hline $14: 0$ & $-0,0104$ & 0,0111 & 0,9906 & $-0,0071$ & 0,0105 & 0,9879 & 0,0024 & 0,0064 & 0,9966 & 0,0018 & 0,0066 & 0,9992 \\
\hline $16: 0$ & $-0,0288$ & 0,0320 & 0,9904 & $-0,0182$ & 0,0301 & 0,9891 & 0,0073 & 0,0188 & 0,9983 & 0,0072 & 0,0193 & 0,9993 \\
\hline $16: 1 \mathrm{n} 7$ & $-0,0307$ & 0,0324 & 0,9905 & $-0,0207$ & 0,0306 & 0,9889 & 0,0059 & 0,0191 & 0,9984 & 0,0070 & 0,0195 & 0,9992 \\
\hline $18: 4 n 3$ & $-0,0085$ & 0,0695 & 0,9895 & $-0,0048$ & 0,0062 & 0,9903 & 0,0009 & 0,0040 & 0,9975 & $-0,0001$ & 0,0040 & 0,9978 \\
\hline $20: 5 n 3$ & $-0,0611$ & 0,0348 & 0,9704 & $-0,0109$ & 0,0259 & 0,9910 & 0,0010 & 0,0173 & 0,9989 & 0,0078 & 0,0189 & 0,9950 \\
\hline $22: 6 n 3$ & $-0,0161$ & 0,0109 & 0,9889 & $-0,0096$ & 0,0098 & 0,9891 & $-0,0027$ & 0,0061 & 0,9940 & $-0,0009$ & 0,0063 & 0,9970 \\
\hline
\end{tabular}

r: Coeficiente de correlación.

w: Biomasa húmeda.

d: Biomasa liofilizada.

Oo: Ordenada en el origen.

P: Pendiente de la regresión lineal: $\mathrm{mg}$ de ácido graso identificado vs $\mathrm{mg}$ de biomasa metilada (este valor corresponde a la fracción másica del peso seco) 


\section{RESULTADOS Y DISCUSION}

\section{a) Estudio de la linealidad}

En las figuras 1 y 2 y en la tabla I se presentan los resultados de los cromatogramas para el método de Lepage y Roy por vía húmeda y liofilizado, con una extracción y con tres extracciones reunidas (modificación propuesta). En la tabla I, aparece la información referente a aquellos ácidos grasos que son mayoritarios (14:0, 16:0, $16: 1 n 7,18: 4 n 3,20: 5 n 3$ y $22: 6 n 3)$ con el objeto de simplificar el tratamiento de datos y obviar los problemas de dispersión de resultados que presentan los ácidos grasos minoritarios, ya que se ven más afectados por los errores experimentales de la integración y por diferentes recuperaciones en la extracción a pesar de duplicar las muestras. La tabla muestra los resultados obtenidos (coeficiente de correlación, ordenada en el origen y pendiente), para el ajuste por regresión lineal de la cantidad de ácido graso

\section{a)}

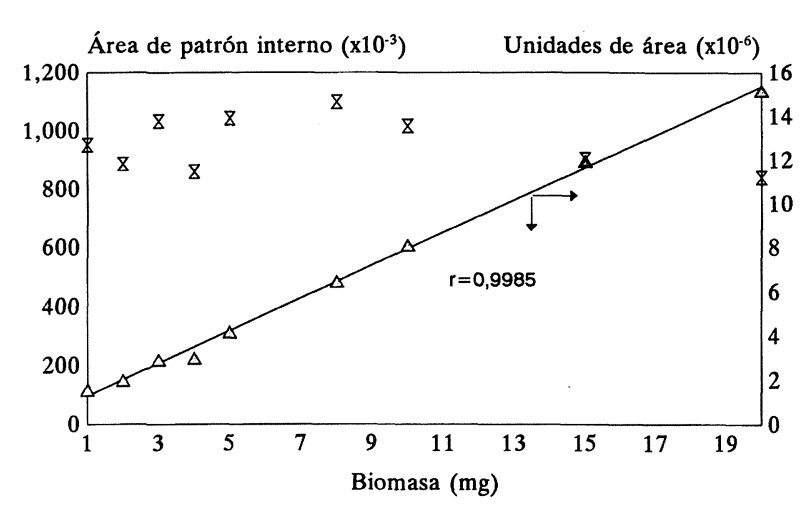

$\triangle$ Área total 8 Área patrón

b)

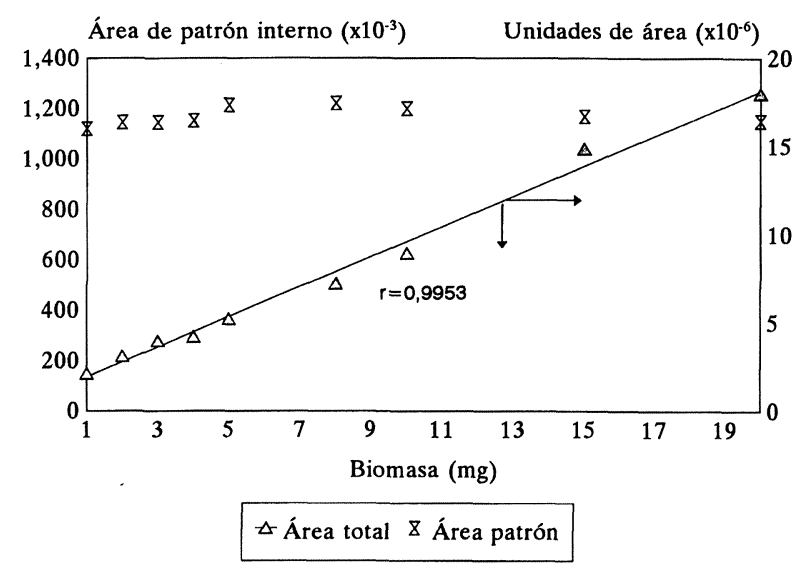

Fig. 1

Efecto de la cantidad de biomasa húmeda en la extracción de ácidos grasos.

a) con $1 \mathrm{ml}$ de hexano.

b) con $3 \mathrm{ml}$ de hexano. a)

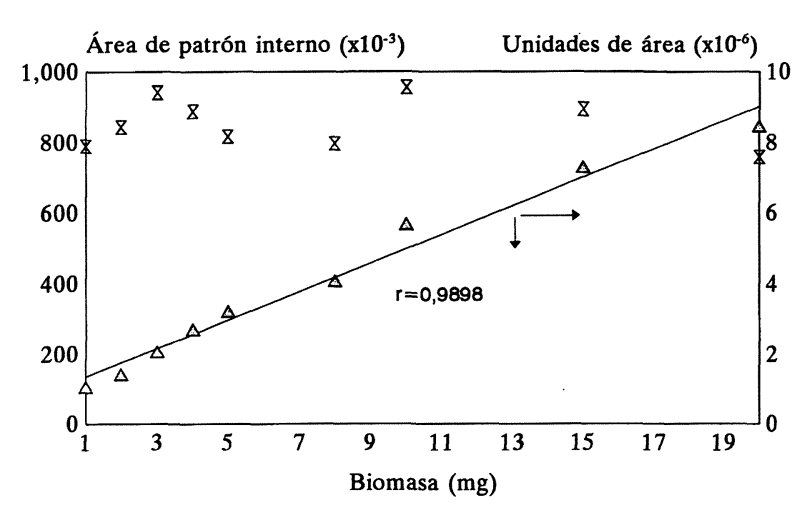

$\triangle$ Área total 8 Área patrón

b)

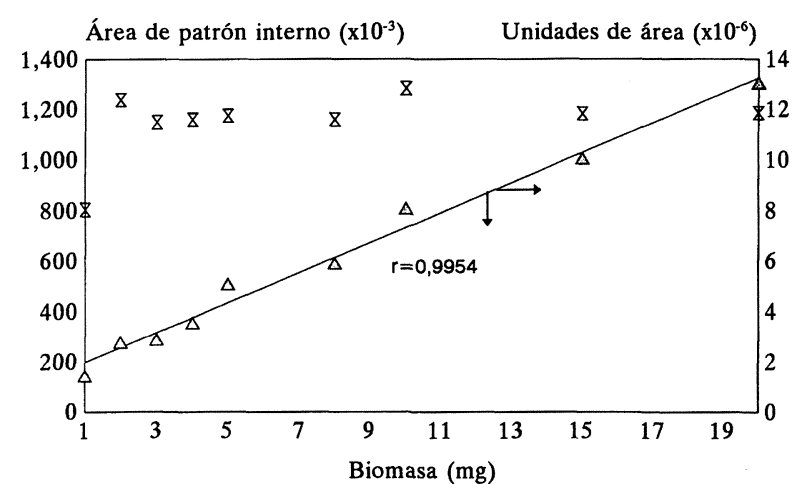

$\triangle$ Área total 8 Área patrón

Fig. 2

Efecto de la cantidad de biomasa liofilizada en la extracción de ácidos grasos.

a) con $1 \mathrm{ml}$ de hexano.

b) con $3 \mathrm{ml}$ de hexano.

identificado, (mg), vs cantidad de biomasa metilada, (mg), para cada uno de los ácidos grasos mencionados anteriormente, diferenciando el estado físico de la biomasa (húmeda o liofilizada) y el volumen de disolvente extractante. Los valores de la tabla indican en todos los casos y para todos los ácidos grasos seleccionados un buen ajuste, observándose una diferencia apreciable entre los procedimientos a partir de liofilizado y biomasa húmeda, como queda reflejado en el análisis multifactorial de la varianza (tabla II), donde el estado físico de la biomasa es un factor estadísticamente significativo.

En las figuras 1 y 2 se ha representado la cantidad total identificada en unidades de área y también la cantidad de patrón interno detectado en cada análisis. Puede apreciarse como la linealidad se mantuvo en todo el intervalo de experimentación. Con estas figuras se pone de manifiesto la bondad del uso de patrón interno como método de integración, así como que la aproximación para el cálculo de los factores de respuesta es aceptable. 
Tabla II

Resultados del ANOVA para los datos de las figuras 3 y 4

\begin{tabular}{|c|c|c|c|c|c|c|c|c|c|c|c|}
\hline & \multicolumn{3}{|c|}{$\begin{array}{l}\text { Estadísticos F-ratio de los } \\
\text { factores que se han tomado como } \\
\text { fuente de variación en el análisis }\end{array}$} & \multicolumn{2}{|c|}{$\begin{array}{c}\text { Estadísticos F-ratio de } \\
\text { las interacciones }\end{array}$} & \multicolumn{6}{|c|}{ Intervalos de confianza para las medidas } \\
\hline & A & B & C & $A C$ & $\mathrm{BC}$ & media global & w & $d$ & $1 \mathrm{ml}$ & $3 \mathrm{ml}$ & $\begin{array}{c}\mathrm{GH} \\
(\mathrm{mg})\end{array}$ \\
\hline $14: 0$ & $97,114^{\star \star}$ & $0,098 \mathrm{~ns}$ & $5,048^{\star \star}$ & $5,448^{\star \star}$ & - & $0,797-0,843$ & $0,892-0,955$ & $0,685-0,747$ & $0,785-0,848$ & $0,792-0,854$ & $3-8$ \\
\hline 16:0 & $192,416^{\star \star}$ & $1,190 \mathrm{~ns}$ & $9,495^{\star \star}$ & $9,003^{\star \star}$ & - & $2,359-2,447$ & $2,628-2,752$ & $2,055-2,178$ & $2,319-2,442$ & $2,364-2,487$ & $4-10$ \\
\hline $16: 1 \mathrm{n} 7$ & $207,062^{\star *}$ & 1,658 ns & $9,251^{\star \star}$ & $10,225^{\star *}$ & - & $2,357-2,441$ & $2,625-2,743$ & $2,055-2,173$ & $2,314-2,433$ & $2,365-2,484$ & $3-10$ \\
\hline $18: 4 n 3$ & $63,405^{\star \star}$ & $0,476 \mathrm{~ns}$ & $2,218 \mathrm{~ns}$ & $3,292^{*}$ & - & $0,453-0,485$ & $0,506-0,552$ & $0,386-0,431$ & $0,452-0,496$ & $0,441-0,486$ & $2-8$ \\
\hline $20: 5 n 3$ & $13,455^{\star \star}$ & $3,813 \mathrm{~ns}$ & $1,532 \mathrm{~ns}$ & - & - & $2,035-2,312$ & $2,224-2,616$ & $1,731-2,122$ & $1,896-2,238$ & $2,108-2,500$ & $4-15$ \\
\hline $22: 6 n 3$ & $247,726^{\star \star}$ & $23,286^{\star \star}$ & $18,951^{\star *}$ & $13,4^{\star \star}$ & $7,315^{\star \star}$ & $0,669-0,695$ & $0,756-0,793$ & $0,571-0,608$ & $0,635-0,672$ & $0,692-0,729$ & $1-10$ \\
\hline
\end{tabular}

** Estadísticamente significativo con un nivel de significación menor de 0,01.

* Estadísticamente significativo con un nivel de significación mayor de 0,01. ns: No significativo.

A: Estado físico de la biomasa.

B: Volumen de extracción.

C: Cantidad de biomasa metilada.
GH: Grupos homogéneos. w: Biomasa húmeda. d: Biomasa liofilizada. b) Comparación del método entre vía húmeda y biomasa liofilizada

En las figuras 3 y 4 se ha representado el contenido en peso seco de cada ácido graso (obtenido de su correspondiente cromatograma, como se ha indicado en el procedimiento experimental), frente a la cantidad de biomasa tratada (liofilizada y húmeda) para la extracción con $1 \mathrm{ml}$ y 3 $\mathrm{ml}$ de hexano, respectivamente. Si consideramos el tipo de biomasa, puede verse que en casi todos los casos la recuperación es mayor cuando se utiliza biomasa húmeda. Esta diferencia puede deberse a la posible aparición de degradaciones enzimáticas y cambios químicos derivados de procesos oxidativos cuando la biomasa es liofilizada (21), que pueden modificar el contenido de la fracción lipídica.

Por otro lado, si comparamos ambas figuras se pone de manifiesto que la extracción en tres etapas es ligeramente más favorable que en una sola, ya que se recupera úna mayor cantidad de ácidos grasos identificados, y es, por tanto, el procedimiento más fiable, porque realiza una extracción completa del analito. Este hecho queda reflejado al' comparar los cromatogramas de tres extracciones consecutivas de la misma muestra analizadas independientemente, observándose que para $10 \mathrm{mg}$ de biomasa metilada o cantidades mayores aún quedaría analito en la tercera extracción

Los datos de las figuras 3 y 4 han sido sometidos a un análisis estadístico multifactorial de la varianza tomando como factores el estado físico de la biomasa, el número de extracciones y la cantidad de biomasa sometida a metilación e incluyendo las interacciones de orden dos entre los mismos. Aquellas interacciones que no resultaron significativas fueron eliminadas del análisis y en la tabla II aparecen los resultados incluyendo las interacciones no rechazadas.
Tabla III

Regresión múltiple de modelo lineal $(Y=C+P * X)$ incluyendo factores cualitativos (X: cantidad de biomasa metilada)

\begin{tabular}{|c|c|c|c|c|}
\hline \multirow{3}{*}{$\begin{array}{l}\text { Acido } \\
\text { graso }\end{array}$} & \multicolumn{2}{|c|}{ Parámetros del modelo } & \multicolumn{2}{|c|}{ Factores cualitativos modificados } \\
\hline & $C(1)$ & $P$ & Biomasa & $3 \mathrm{ml} \mathrm{de}$ \\
\hline & & & liofilizada & hexano \\
\hline 14:0 & $0,914^{\star *}$ & $0,000747 \mathrm{~ns}$ & $-0,2071^{\star *}$ & $0,0066 \mathrm{~ns}$ \\
\hline $16: 0$ & $2,668^{\star \star}$ & $-0,000052 \mathrm{~ns}$ & $-0,5736^{\star \star}$ & $0,0451 \mathrm{~ns}$ \\
\hline $16: \ln 7$ & $2,63 \cdot 1^{\star *}$ & 0,00374 ns & $-0,5704^{\star \star}$ & $0,0511 \mathrm{~ns}$ \\
\hline $18: 4 n 3$ & $0,503^{\star \star}$ & $0,00407^{\star}$ & $-0,1203^{\star \star}$ & $-0,0104 \mathrm{~ns}$ \\
\hline $20: 5 n 3$ & $2,220^{\star *}$ & $0,00905 \mathrm{~ns}$ & $-0,4933^{\star *}$ & $0,2626 \mathrm{~ns}$ \\
\hline $22: 6 n 3$ & $0,667^{\star \star}$ & $0,01049^{\star \star}$ & $-0,1851^{\star *}$ & $0,0567 \mathrm{~ns}$ \\
\hline
\end{tabular}

** Estadísticamente significativo con un nivel de significación menor de 0,01 .

* Estadísticamente significativo con un nivel de significación mayor de 0,01 .

ns: No significativo.

(1) Valores iniciales de los factores cualitativos (biomasa húmeda y $1 \mathrm{ml}$ de volumen para extracción).

También se ha hecho una regresión múltiple con modelo lineal en función de la cantidad metilada (tabla III), incluyendo factores cualitativos (estado físico de la biomasa y volumen de extracción). Ambos tratamientos con un nivel de confianza del $95 \%$. Estos resultados ponen de manifiesto que el estado físico (liofilizado o húmedo) es determinante en el resultado del análisis, ya que este factor ha sido significativo en los ácidos estudiados en todos los casos, como refleja su estadístico F-ratio (columna A de la tabla II).

La interpretación de los datos del modelo de regresión lineal (tabia III) se puede ver con un ejemplo: la ecuación 


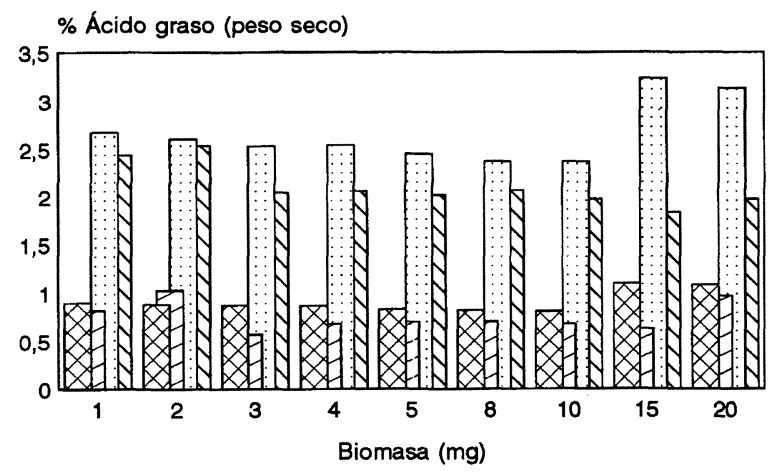

14:0 (bio.húmeda+1 ml) $\Xi_{14: 0}$ (liof.+1 ml) $\square_{16: 0}$ (bio.húmeda+1 ml) $\nabla_{16: 0}$ (liof.+1 ml)

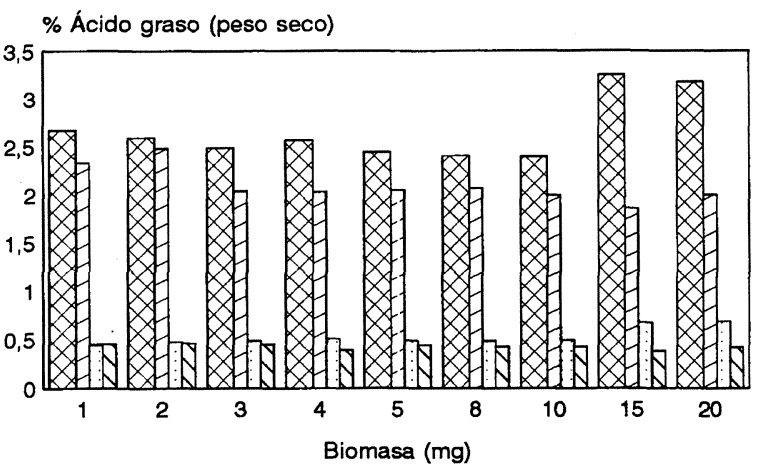

16:1n7 (bio.húm.+1 ml) छ 16:1n7 (liof. +1 ml) $\square_{18: 4 n 3}$ (bio.húm. +1 ml) $\nabla_{18: 4 n 3}$ (liof. +1 ml)

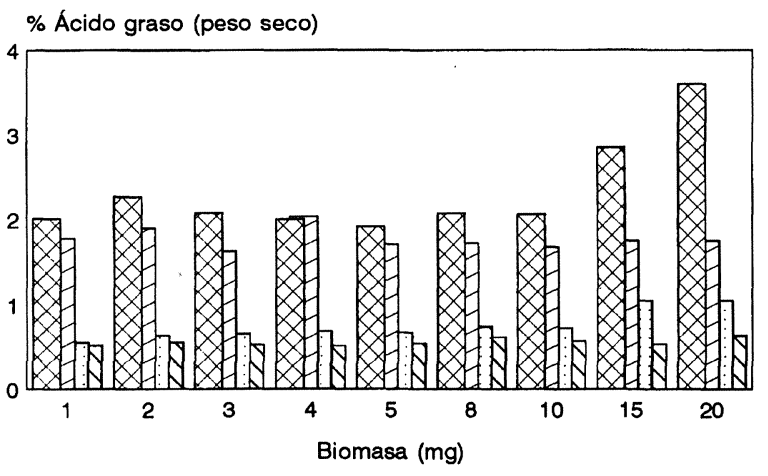

$\bigotimes_{20: 5 \mathrm{n} 3}$ (bio.hum. +1 ml) 曰20:5n3 (liof. $+1 \mathrm{ml}$ ) $\square_{22: 6 n 3}$ (bio.hum.+1 ml) $\nabla_{22: 6 \mathrm{n3}}$ (liof.+1 ml)

Fig. 3

Influencia del estado físico de la biomasa en la extracción de los ésteres metílicos con $1 \mathrm{ml}$ de hexano.

de regresión es $Y=C+P{ }^{*} X$, donde $Y$ es la variable dependiente $o$ la respuesta experimental, es decir, el porcentaje sobre peso seco de cada ácido graso; $X$ es la cantidad de biomasa metilada en mg y $P$ y $C$ son los parámetros del modelo, pendiente y ordenada en el origen, respectivamente. El valor de la constante, $\mathrm{C}$, corresponde a

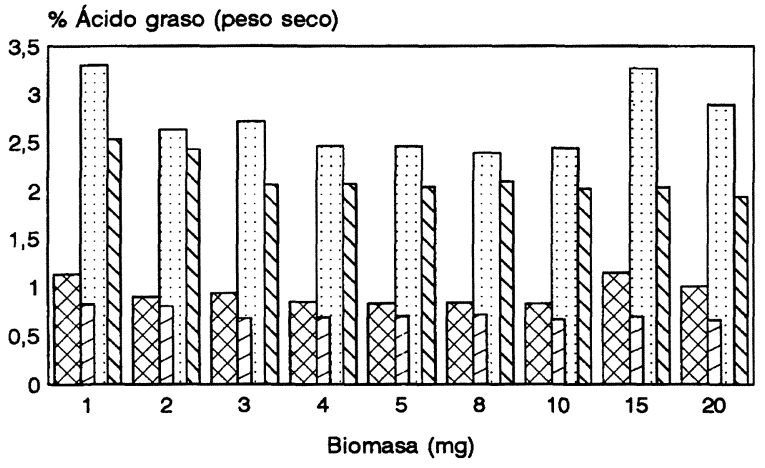

14:0 (bio.húmeda+3 ml) 曰14:0 (liof.+3 ml) $\square$ 16:0 (bio.húmeda +3 m) $\nabla_{16: 0}$ (liof. +3 ml)

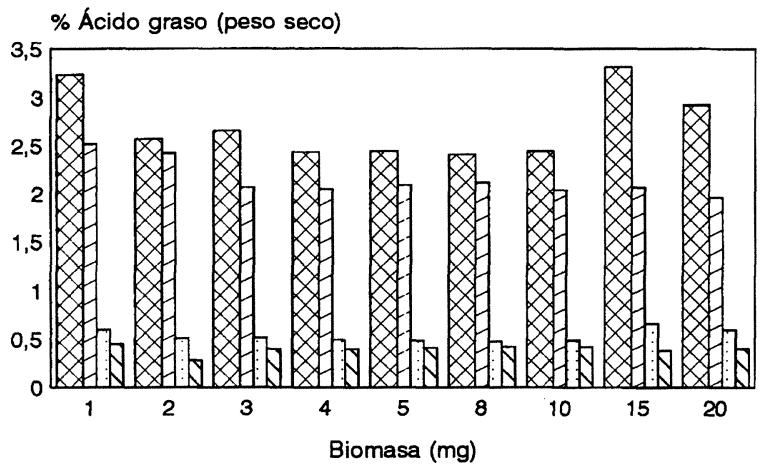

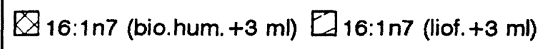

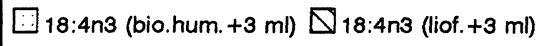

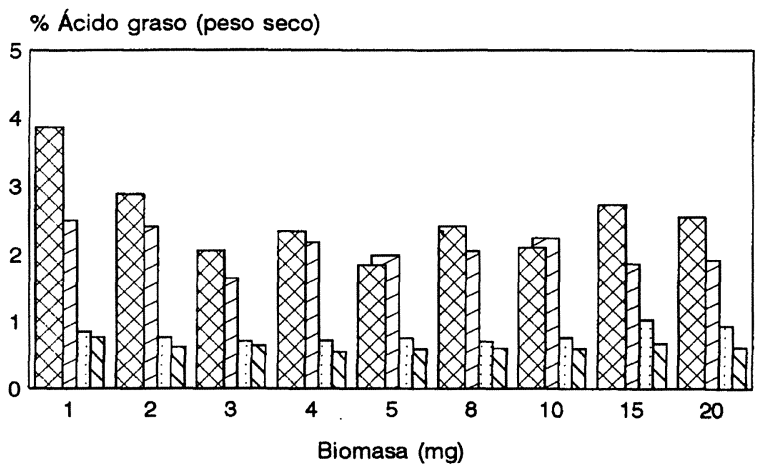

$\bigotimes_{20: 5 \mathrm{n} 3}$ (bio. hum+3 ml) $\square_{20: 5 \mathrm{n} 3}$ (liof.+3 ml)

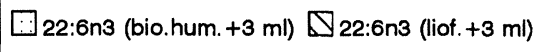

Fig. 4

Influencia del estado físico de la biomasa en la extracción de los ésteres metílicos con $3 \mathrm{ml}$ de hexano.

los valores iniciales de la variable cualitativa: biomasa húmeda como estado físico y $1 \mathrm{ml}$ de hexano como volumen extractante. Este valor se modifica para un cambio de estado físico o de volumen, aplicando los coeficientes para liofilizado y $3 \mathrm{ml}$ de volumen de extracción (columnas 3 y 4 tabla III). Así, para el caso del análisis con 
$8 \mathrm{mg}$ de biomasa y el ácido eicosapentaenoico los resultados serían los siguientes:

- Biomasa húmeda y $1 \mathrm{ml}$ de hexano: \% en peso de $E P A=2,220+0,00905 * 8=2,292$. La medida experimental fue 2,071.

- Biomasa húmeda y $3 \mathrm{ml}$ de hexano: \% en peso seco de $E P A=2,220+0,2626+0,00905 * 8=2,555$. La medida experimental fue 2,410 .

- Liofilizado y $1 \mathrm{ml}$ de hexano: \% en peso seco de EPA $=2,220+(-0,4933)+0,00905 * 8=1,799$. La medida experimental fue 1,719 .

- Liofilizado y $3 \mathrm{ml}$ de hexano: \% en peso seco de EPA $=2,220+0,2626+(-0,4933)+0,00905{ }^{*} 8=2,062$. La medida experimental fue 2,043 .

El hecho de obtener el modelo de regresión no tiene, lógicamente, fines predictivos y se ha efectuado como otro tratamiento alternativo para apoyar las conclusiones que se derivan del análisis de la varianza. Como se puede ver en la tabla III, los coeficientes para el factor biomasa liofilizada son todos estadísticamente significativos y de valor negativo, lo que conduce a obtener menores cantidades identificadas para la biomasa liofilizada, coincidiendo con los estadísticos F-ratio para el mismo factor en el análisis de la varianza (tabla II, columna A), que discrimina significativamente las medias para las dos condiciones experimentales del estado físico. Por otra parte, la ordenada en el origen de la regresión múltiple, $\mathrm{C}$, es estadísticamente significativa para todos los ácidos grasós estudiados, y su valor se encuentra dentro del intervalo de confianza para las medias obtenidas a partir de biomasa húmeda, que es el factor cualitativo de referencia para el estado físico en este tratamiento.

En cuanto a la cantidad de biomasa metilada, a pesar de presentar un buen ajuste para la linealidad (tabla I, figuras 1 y 2), aparece como un factor influyente en el análisis de la varianza, siendo significativo para cuatro de los ácidos grasos estudiados. A consecuencia de este hecho se ha incluido en la tabla II el intervalo de metilación que el análisis de la varianza da como grupos homogéneos (aquellos valores del intervalo experimental para los cuales no hay diferencias entre sus medias que sean debidas a los factores que se han considerado influyentes en el resultado), incluyendo en la tabla el grupo mayoritario (columna GH). De esta forma, y para obtener una mayor fiabilidad en los resultados, se recomienda utilizar dichos intervalos para la realización de los análisis.

Respecto al factor volumen extractante, ninguno de los dos tratamientos lo da como estadísticamente significativo, debido, posiblemente, a que la relación de extracción patrón/ácido graso es constante, y esto hace que la relación para la integración no tenga una modificación diferenciada en el estudio de las medias para este tratamiento. Es decir, que a pesar de extraer menor cantidad en valor absoluto con $1 \mathrm{ml}$ de hexano, se mantiene prácticamente constante la relación área ácido graso/área patrón interno, que es la referencia para la integración.

Como conclusión de este trabajo, reiteramos la conveniencia de realizar los análisis a partir de biomasa húmeda como hecho relevante, la utilización de los intervalos de metilación antes mencionados y aconsejamos realizar tres extracciones con $1 \mathrm{ml}$ de hexano, salvando el error experimental que se ha podido cometer en este estudio.

\section{BIBLIOGRAFIA}

1. Yongmanitchai, W. y Ward, O. P. $-« W-3$ fatty acids. Alternative sources of production».-Process. Biochem. 24 (1989) 117-125.

2. Borowitzka, M. A.-«Fats, oils and hydrocarbons" en «Micro-algal biotechnology".-M. A. Borowitzka y L. J. Borowitzka (Eds).-Cambridge University Press, Cambridge, p. 257 (1988).

3. Roessler, P. G.-«Environmental control of glycerolipid metabolism in microalgae: Commercial implications and future research directions».-J. Phycol. 26 (1990) 393-399.

4. López Alonso, D.; Molina Grima, E.; Sánchez Pérez, J. A.; García Sánchez, J. L. y García Camacho, F.-«Isolation of clones of Isochrysis galbana rich in eicosapentaenoic acid"-Aquaculture 102(1992a) 363-371.

5. López Alonso, D.; Molina Grima, E.; Sánchez Pérez, J. A.; García Sánchez, J. L. y García Camacho, F.-«Fatty acid variation among different isolates of a single strain of Isochrysis galbana".-Phytochemistry 31 (1992b) 3901-3904.

6. Alvarez Covelas, M.- «Lipids in microalgae. A review II. Environment».-Grasas y Aceites 40 (1989) 213-223.

7. Napolitano, G. y Shantha, N. C.- «Gas chromatography of fatty acids"-Journal Chromatography 624 (1992) 37-51.

8. Napolitano, G.; Ackman, R. y Ratnayake, W. M. N.-«Fatty acid composition of three cultured algal species (Isochrysis galbana, Chaetoceros gracilis y Chaetoceros calcitrans) used as food for bivalve larvae".-Journal World Aquaculture Society 21 (1990) 122-130.

9. Sukenik, A. y Livne, A.- «Variations in lipid and fatty acid content in relation to acetyl CoA carboxylase in the marine prymnesiophyte Isochrysis galbana».-Plant Cell Physiol. 32 (1991) 371-378.

10. Renaud, S. M.; Parry, D. L.; Luong-Van, T.; Kuo, C.; Padovan, A., y Sammy, N.-«Effect of light intensity on the proximate biochemical and fatty acid composition of Isochrysis sp and Nannochloropsis oculata for the use in tropical aquaculture".-Journal Applied Phycology 3 (1991) 45-53.

11. Flynn, K. J.; Garrido, J. L.; Zapata, M.; Öpik, H., y Hipkin, C R. "Changes in fatty acids, amino acids and carbon/nitrogen biomass during nitrogen starvation of ammonium -and nitrate- grown /sochrysis galbana».-Journal Applied Phycology 4 (1992) 95-104.

12. Ahlgren, G., y Merino, L.-«Lipid analysis of freshwater microalgae: A method study"-Arch. Hydrobiol. 121 (1991) 295-306.

13. Whyte, J. N. C.- «Fatty acid profiles from direct methanolysis of lipids in tissue of cultured species».-Aquaculture 75 (1988) 193-203.

14. Lepage, G. y Roy, C. C.- «Improved recovery of fatty acid through direct transterification without prior extraction of purification».-J. Lipid Res. 25 (1984) 1391-1396.

15. Lepage, G. y Roy, C. C.-«Direct transesterification of all classes of lipids in a one-step reaction".-J. Lipid Res. 27 (1986) 114-120.

16. García Camacho, F.; Molina Grima, E.; Martínez Sancho, $M^{a}$ E. y Sánchez Villasclaras, S.-«Determinación de ácidos grasos en microalgas marinas. Comparación de diversos métodos de extracción lipídica".-Grasas y Aceites 41 (1990) 13-18.

17. Molina Grima, E.; Sánchez Pérez, J. A.; García Sánchez, J. L.; García Camacho, F. y López Alonso, D.- «EPA from Isochrysis galbana. Growth conditions and productivity».-Process. Biochem. 27 (1992) 299Grow.

18. Molina Grima, E.; Sánchez Pérez, J. A.; García Camacho, F.; García Sánchez, J. L. y López Alonso, D.-«n-3 PUFA productivity in chemostat cultures of microalgae».-Appl. Microbiol. Biotechnol. 38 (1993) 599-605.

19. Sánchez Pérez, J. A.-«Productividad en ácidos grasos poliinsaturados $n-3$ de la microalga marina Isochrysis galbana. Condiciones de crecimiento y selección fenotípica».-Tesis Doctoral. Universidad de Granada (1992) p. 312

20. Molina Grima, E.; Martínez Sancho, M ${ }^{\mathrm{a}}$ E.; Sánchez Villasclaras, S.; García Camacho, F. y Contreras Gómez, A.-«Growth and biochemical composition with emphasis on the fatty acids of Tetraselmis sp».Appl. Microbiol. Biotechnol. 36 (1991) 21-25.

21. Kates, M.-«Lipid extraction procedures: extraction of microorganism», en «Techniques of lipidology. Isolation, analysis and identification of lipids».-2nd Edition.-Burdon, R. H. y Knippenberg, P. H. (Eds).-Elsevier Science Publishers, Amsterdam, p. 106 (1988).

(Recibido: Julio 1993) 